\title{
Comparative Study of SWT-SVD and DWT-SVD Digital Image Watermarking Technique
}

\author{
Neha R. Sawant \\ Department of Electronics \\ SSVPS's B.S.D COE, Dhule \\ Maharashtra, India
}

\author{
Pravin S. Patil \\ Department of Electronics \\ SSVPS's B.S.D COE, Dhule \\ Maharashtra, India
}

\begin{abstract}
Due to use of the latest computer technology in early days with wide available tools with various advance application, it is very easy for the unknown users to produce illegal copies of multimedia data which are floating across the Internet. To protect multimedia data such as images, videos,etc. on the Internet many techniques are available including various encryption techniques, steganography techniques, watermarking techniques and information hiding techniques. Digital watermarking is a technique in which a piece of digital information is embedded into a cover image and extracted later for ownership verification. Secret digital data which is hidden can be embedded either in spatial domain or in frequency domain of the cover data. in this paper frequency domain technique is used.by using singular value decomposition (SVD) with existing method DWT (Discrete Wavelet transform) that is DWT-SVD Combine watermarking technique and proposed method includes stationary wavelet transformation (SWT) with SVD that is SWT-SVD based water marking technique is proposed for hiding watermark. The quality of the watermarked image and extracted watermark is measured using peak signal to noise ratio (PSNR). A user defined or predefined watermark can be embedded within the image without disturbing quality of the image. It is observed that the quality of the watermarked image is maintained of proposed method results are tested for various attacks which include Salt and Pepper noise, Gaussian noise, cropping and compression, rotation etc. for both DWT and SWT for high. Robustness. A large payload can also be embedded in this proposed algorithm.SWT-SVD result PSNR is get improved as compare to DWT-SVD.In this paper Both the Methods are Implemented by Using MATLAB and Comparative Experimental Results are Reported.
\end{abstract}

\section{Keywords}

Watermarking, Stationary wavelet transformation (SWT), Singular Value Decomposition (SVD), Discrete Wavelet Transform (DWT), MSE (mean square error), PSNR (peak signal to noise ratio), large payload, Robustness

\section{INTRODUCTION}

In this paper, digital watermarking is process of an Embedding piece of code in digital data image and in the cover data image which is to be protected from duplication and extracted later for ownership verification in security aspects these are the main important applications of digital watermarking. The major point of digital watermarking is to find the balance among the aspects such as robustness to various attacks, security and invisibility. Property of Robustness and Fragility are important for ownership verification and image authentication respectively in this paper by using DWT-SVD and SWT-SVD with the help of PSNR Values comparative analysis is done. SVD along with
DWT is existing method and SVD along with SWT is the proposed method, after study analysis with different cover and logo images for both methods it founds that SWT-SVD gives better PSNR values result than DWT-SVD domain digital image watermarking. Theincreasing perceptibility will also decrease the quality ofwatermarked image. Generally watermark could not directly hidden, it is done by modifying the intensity value or pixel value of an image (spatial domain)or its frequency components. The former technique which is used for watermarking is spatial domain technique and frequency domaintechnique. After applying transforms it is converted into different sub components as $\mathrm{HH}, \mathrm{HL}, \mathrm{LH}$, and LL, in which high frequency components $\mathrm{HH}$ are affected by most of the signal processing techniques such as lossy compression, so in order to increase the robustness, ideally the watermark is preferred to be placed in the low frequency components. But our human visual system is very sensitive to changes in low frequency range. So, i DWT-based watermarking techniques, the DWT coefficients LH,HL and $\mathrm{HH}$ are modified to watermark data. Because of the conflict between robustness and transparency, the modification is usually made in HL, LH and HH sub-bands to maintain better image quality as $\mathrm{HH}$ band contains finer details and contribute insignificantly towards signal energy. Hence, watermarking embedding in this region will not affect the perpetual fidelity of the cover image.By considering SWT-SVD combined full band watermarking is possible.in DWT-SVD technique to embed watermark image into the main or cover image, which proves robust to various kind of attacks which are mentioned as SWT helps to increase the payload i.e., large size watermark, which is an advantage over DWT-SVD techniques.In this paper with the help of PSNR Values[4] Comparative analysis is done. SWT-SVD gives better result than DWT-SVD domain digital image watermarking.

\section{OWERVIEW OF DWT-SVD}

\subsection{DWT (Discrete Wavelet Transform)}

The DWT decomposes input image into four components namely LL(low pass frequency) operation to the rows, LH(vertical high), HL(horizontal high) and HH(high pass frequency)Operation to the columns .which is shown in Fig.1.

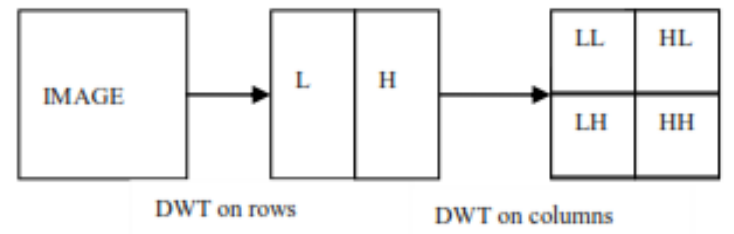

Fig 1. DWT Decomposition of Image 
The lowest resolution level LL consists of the approximation part of the original image. The remaining three resolution levels consist of the detail parts and give the vertical high (LH), horizontal high (HL) and high (HH)frequencies. In the proposed algorithm, watermark is embedded into the host image (cover image) by modifying the coefficients of highfrequency bands i.e. HH sub-band. For a one level decomposition, the discrete two-dimensional wavelet transform of the image function $\mathrm{f}(\mathrm{x}, \mathrm{y})$ can be written as

$$
\begin{aligned}
& \mathrm{LL}=\left[\left(\mathrm{f}(\mathrm{x}, \mathrm{y})^{\star} \emptyset(-x) \emptyset(-y)\right)(2 \mathrm{n}, 2 \mathrm{~m})\right]_{(\mathrm{n}, \mathrm{m}) \mathrm{ez}^{2}} \\
& \mathrm{LH}=\left[\left(\mathrm{f}(\mathrm{x}, \mathrm{y})^{\star} \emptyset(-x) \Psi(-y)\right)(2 \mathrm{n}, 2 \mathrm{~m})\right]_{(\mathrm{n}, \mathrm{m}) \mathrm{e} z^{2}} \\
& \mathrm{HL}=\left[\left(\mathrm{f}(\mathrm{x}, \mathrm{y})^{\star} \Psi(-x) \emptyset(-y)\right)(2 \mathrm{n}, 2 \mathrm{~m})\right]_{(\mathrm{n}, \mathrm{m}) \mathrm{e} z^{2}} \\
& \mathrm{LL}=\left[\left(\mathrm{f}(\mathrm{x}, \mathrm{y})^{\star} \Psi(-x) \emptyset(-y)\right)(2 \mathrm{n}, 2 \mathrm{~m})\right](\mathrm{n}, \mathrm{m}) \mathrm{e} \mathrm{z}^{2}
\end{aligned}
$$

Where, $\phi(\mathrm{t})$ is a low pass scaling function and $\psi(\mathrm{t})$ is the associated band pass wavelet function.

\subsection{SVD (singular value decomposition)}

SVD is special matrix transform.it includes numbers with intrinsic characteristics. SVD provides excellent stability which prevents remarkable big changes due to small image disturbance hence SVD is widely used.SVD transform decomposes (SVD) is a factorization of a real or complex matrix, with many useful applications in signal processing and statistics. The singular value decomposition of an $\mathrm{M} \times \mathrm{N}$ real or complex matrix $\mathrm{M}$ is a factorization of the form as follows,

$$
\mathrm{M}=\mathrm{U} \Sigma \mathrm{V}^{*}
$$

Where $\mathrm{U}$ is an $\mathrm{M} \times \mathrm{M}$ real or complex unitary matrix, $\Sigma \mathrm{V}$ is an $\mathrm{M} \times \mathrm{N}$ rectangular diagonal matrix with nonnegative real numbers on the diagonal, and $\mathrm{V} *$ is an $\mathrm{N} \times \mathrm{N}$ real or complex unity matrix. Any $M \times N(M \geq N)$ real matrix $A$, can be written as, for $(1 \leq \mathrm{i} \leq \mathrm{N})$,

$$
\mathrm{A}=\mathrm{US} V^{T}=\sum_{i=1}^{N} S_{i} U_{i} V_{i}^{T}
$$

Where $\mathrm{U}$ and $\mathrm{V}$ are orthogonal matrices, and $\mathrm{S}$ is an $\mathrm{M} \times \mathrm{N}$

matrix with the diagonal elements Si representing the singular values of $\mathrm{A}$. $\mathrm{Ui}$ is the ithcolumn vector of $\mathrm{U}, \mathrm{Vi}$ is the ith column vector of $\mathrm{V}$. Ui, $\mathrm{Vi}$ are called left and right singular vectors of A respectively. S has the structure of-

$$
\begin{array}{ccccc} 
& S= & S 1 & & \\
& 0 & & \\
& S 1= & 0 & 0 & 0 \\
& 0 & S 2 & 0 & 0 \\
\vdots & \vdots & \vdots & \vdots \\
0 & 0 & 0 & S 8
\end{array}
$$

To solve many mathematical problems in a linear algebra Singular value decomposition (SVD) tool technique is used. The theoretical background of SVD technique in image processing applications to be noticed is: a) The SVs (Singular Values) of an image has very good stability, which means that when a small value is added to an image, this does not affect the quality with great variation.

b) SVD is able to efficiently represent the intrinsic algebraic properties of an image, where singular values correspond to the brightness of the image and singular vectors reflect geometry characteristics of the image.

c) An image matrix has many small singular values compared with the first singular value. Even ignoring these small singular values in the reconstruction of the image does not affect the quality of the reconstructed image with less loss.

So SVD technique can be applied to any kind of images. If it is a gray-scale image, the matrix values are considered as intensity values and it could be modified directly or changes could be done after transforming images into frequency domain. In SVD after applying transforms it decompose the given matrix into three matrices of same size using orthogonal transform, for decompose this matrix using SVD technique square matrix is always not needed.

\subsection{DWT-SVD Watermarking Scheme}

In this paper for comparative analysis three different input images and 3 different logo images are consider .the result of performance is given on the basis of PSNR values obtained.

Fig 2 gives block diagram of DWT-SVD watermarking.

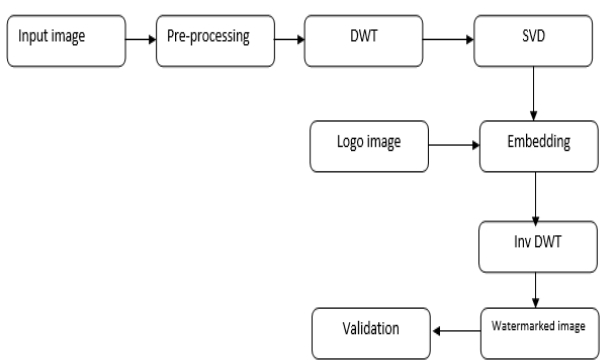

Fig.2 block diagram of DWT-SVD Watermark Embedding

1) In this by using existing method we decompose the cover image into 4sub-bands. It uses one level Haartransformation for decomposition of cover image A into 4 sub-bands.

2) After performing DWT, we perform SVD to eachsub-band images.

i.e, $\mathrm{A}^{\mathrm{K}}=\mathrm{U}_{\mathrm{a}}{ }^{\mathrm{K}} \mathrm{S}_{\mathrm{a}}{ }^{\mathrm{K}} \mathrm{V}_{\mathrm{a}}{ }^{\mathrm{KT}}, \mathrm{k}=1,2,3,4$

Where $\mathrm{k}$ denotes LL, LH, HL and HH sub-bands and $\lambda_{\mathrm{i}}^{\mathrm{K}}{ }_{\mathrm{i}}=1$, $\mathrm{n}$ denotes the singular values of $\mathrm{S}_{\mathrm{a}}{ }^{\mathrm{K}}$.

3)In the same way, we apply SVD to watermark image,

i.e. $\mathrm{W}=\mathrm{U}_{\mathrm{W}} \mathrm{S}_{\mathrm{W}} \mathrm{V}_{\mathrm{W}}{ }^{\mathrm{T}}$ where $\lambda_{\mathrm{Wi}}, \mathrm{i}=1, \mathrm{n}$ Denotes the singular values of $\mathrm{Sw}$.

4) After this, we modify the singular values of cover image in each sub-band with the singular values of watermark image,

i.e. $\lambda \mathrm{i}^{* \mathrm{~K}}=\lambda \mathrm{i}^{\mathrm{K}}+\alpha \mathrm{k} \lambda \mathrm{wi}$

where, $\mathrm{i}=1, \mathrm{n}$ and $\mathrm{k}=1,2,3$, 4 . 
5) So, we obtain 4 sets of modified DWT coefficients, i.e. $\mathrm{A}^{* \mathrm{~K}}=\mathrm{Ua}^{\mathrm{K}} \mathrm{Sa}^{*}{ }^{\mathrm{K}} \mathrm{Va}^{\mathrm{KT}}$ where $\mathrm{k}=1,2,3,4$

Obtain the watermarked image Aw by performing the IDWT using these 4 modified sub-bands.

\section{Watermark extraction}

1) First of all, we use one-level Haar Transform DWT to decompose watermarked (possibly distorted due to various kinds of attacks) image $A * k$ into 4 sub-bands.

2) Then, we apply SVD to each sub-band, i.e. $\mathrm{A}^{* \mathrm{~K}}=\mathrm{Ua}^{\mathrm{K}} \mathrm{Sa}^{*}$ ${ }^{\mathrm{K}} \mathrm{Va}^{\mathrm{KT}} \mathrm{k}=1,2,3$, where $\mathrm{k}$ denotes the attacked sub-band.

3)Then, we extract the singular values from each sub-band, i.e.

$\lambda w \mathrm{i}^{\mathrm{K}}=\left(\lambda \mathrm{i}^{*}{ }^{\mathrm{K}}-\lambda \mathrm{i}^{\mathrm{K}}\right) / \alpha \mathrm{k}$ where $\mathrm{i}=1, \ldots, \mathrm{n}$ and $\mathrm{k}=1,2,3,4$.

4) Construct the four visual watermarks using the singular vectors, i.e.

$\mathrm{W}^{\mathrm{K}}=\mathrm{UwSwVw}^{\mathrm{T}} \mathrm{k}=1,2,3,4$

\section{SWT}

SWT (Stationary Wavelet transform): SWT is preferred as the wavelet transformation, since unlike the other wavelet transforms, the SWT procedures does not include any down sampling steps, instead, a null placing procedure is applied, Length as the original sequence. Instead, filters are modified at each level, by padding them with zeros as shown Fig.3

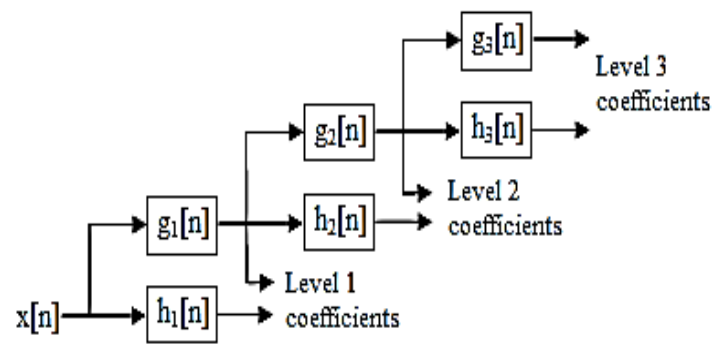

Fig.3.A 3 level SWT filter bank

\subsection{Combine SWT-SVD \\ Embedding process}

The block diagram for embedding watermark using SWTSVD technique is shown in Figure 4.Original image transformed by SWT, which performs multilevel 2-D stationary wavelet decomposition and produces four 3-D arrays namely LL, LH, HL, and $\mathrm{HH}$ which contains the coefficients. Array $\mathrm{HH}$, singular value decomposed and returns a vector of singular values. Similarly, the watermark is also gone through the same process. The watermarked image is obtained by applying ISWT to these coefficients.

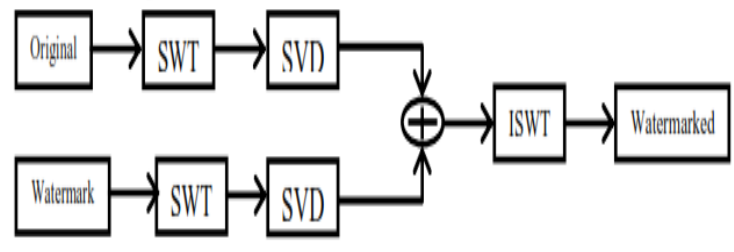

Fig 4.Watermark Embedding Process

\section{- Algorithm for embedding process}

Step 1: Stationary wavelet transformation technique applied to original host image, input is transformed into four 3-D arrays namely LL, LH, HL, HH.

Step 2: SVD technique is applied to high frequency component $\mathrm{HH}$, and the result is a vector of singular values.

Step 3: Same procedure applied to watermark image also.

Step 4: Diagonal matrices of both cover image and watermark image are added with scaling factor.

Step 5: Inverse SWT applied to result to get the embedded watermarked image.

\subsubsection{Extraction process}

The block diagram for extracting watermark using SWT-SVD technique is shown in Figure 5 SWD- SVD, ISWT transformations applied in same order to get watermark from the watermarked image.

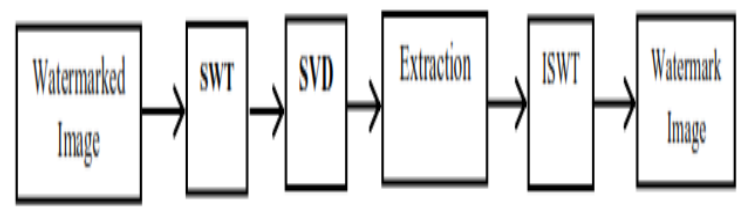

Fig.5.Watermark extraction process

\section{- Algorithm for extraction process}

Step 1: Stationary wavelet transformation technique is applied to watermarked image, input is transformed into four 3-D arrays namely LL, LH, HL, HH.

Step 2: SVD technique is applied to high frequency component $\mathrm{HH}$, and the result is a vector of singular values. Step 3: Watermark image components extracted from SVD transformed image by using same scaling factor.

Step 4: Inverse SWT applied to result to get the retrieved watermark image 


\section{FLOW DIAGRAM}

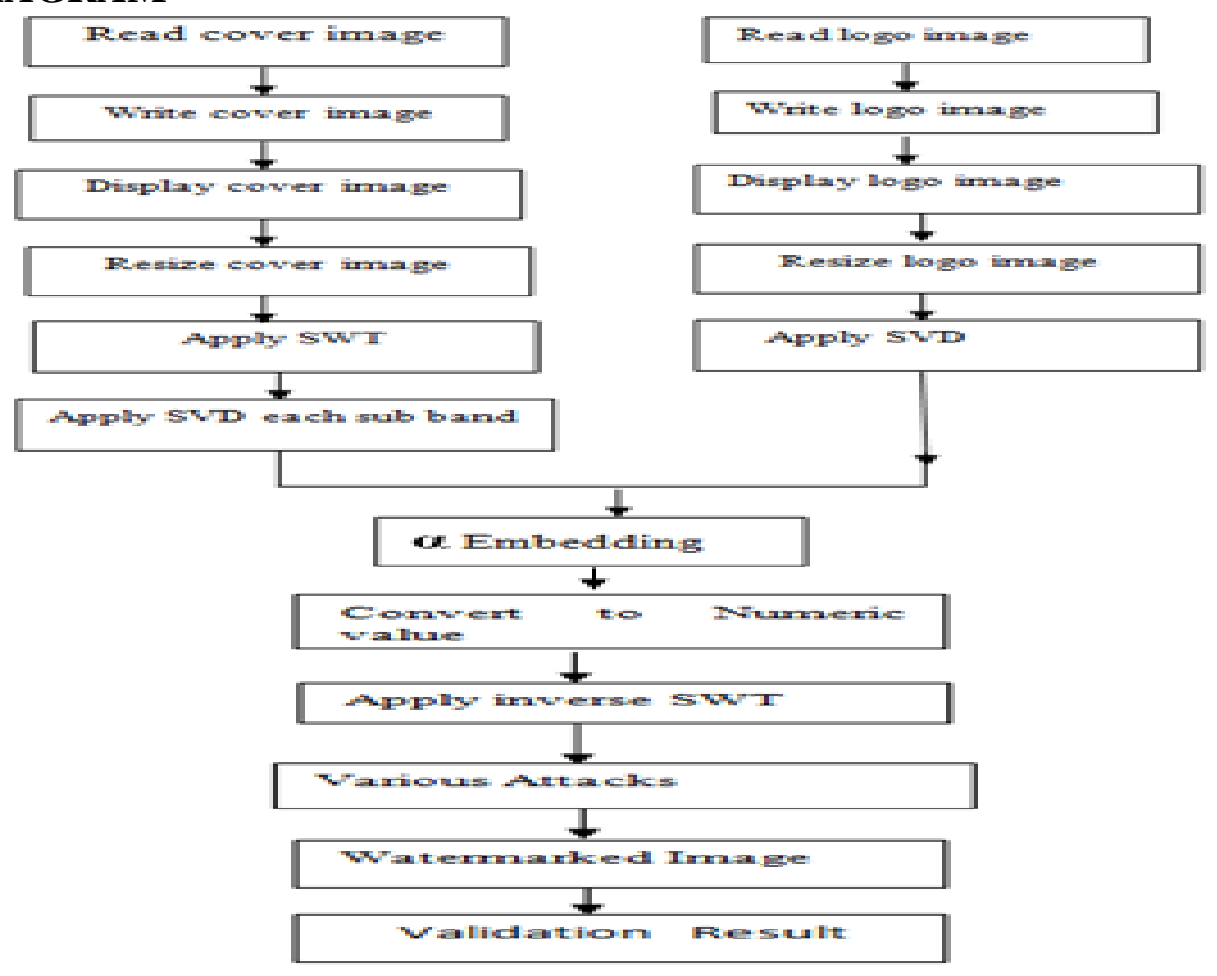

Fig.6 Basic Flow SWT-SVD

\section{Flow/Steps}

Select cover image then Select logo image. Resize both the selected images. Convert to gray scale. Apply SWT and SVD.Embedding of Watermark then Extraction of watermark considering variousattacks Find, PSNR, MSE

\section{EXPERIMENTAL RESULTS}

Considering lena image $\mathrm{n}$ logo image as input and cover image. The DWT-SVD and SWT-SVD both results are given bellow:

The magnitudes of the singular values for each sub-band of the Lena image are shown in the fig.7. Below Figure shows $512 \times 512$ gray scale cover image Lena, the $256 \times 256$ gray scale visual watermark copyright, the watermarked image, and the watermarks constructed from the four sub-bands. The scaling factor i.e. $\mathrm{k}$ for $\mathrm{LL}$ sub-band is taken to be 0.01 and 0.05 for other three sub-bands.Our implemented scheme is based on the idea of replacing singular values of the $\mathrm{HH}$ band with the singular values of watermark. In maximum and minimum singular values of all sub-bands of original image Lena are given by using matlab. The wavelet coefficients are found to have largest value in LL band and lowest for $\mathrm{HH}$ band.

Basically in this work we created such a system that gives us proper results which done in the input image and for that we used different images and calculated the PSNR of this particular code using SWT -SVD and which precisely gives better results than DWT-SVD watermarking made. We used MATLAB software for coding purpose.

Case A) input lena image as cover image and logo image as watermark image the matlab results are as

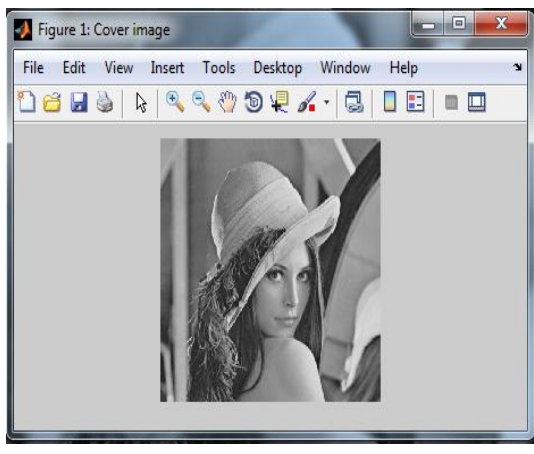

1) Cover Image

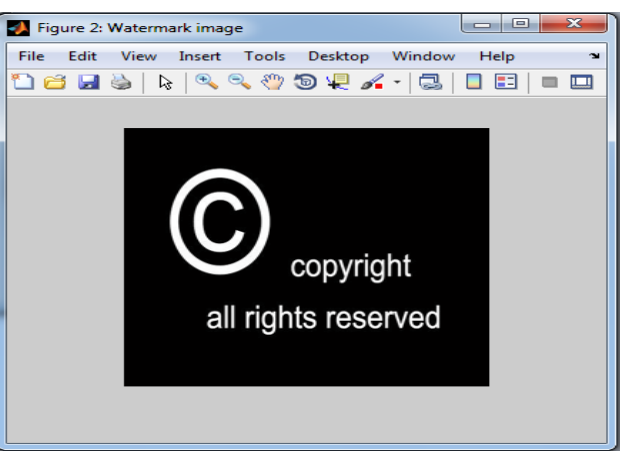

2) Watermark Image 


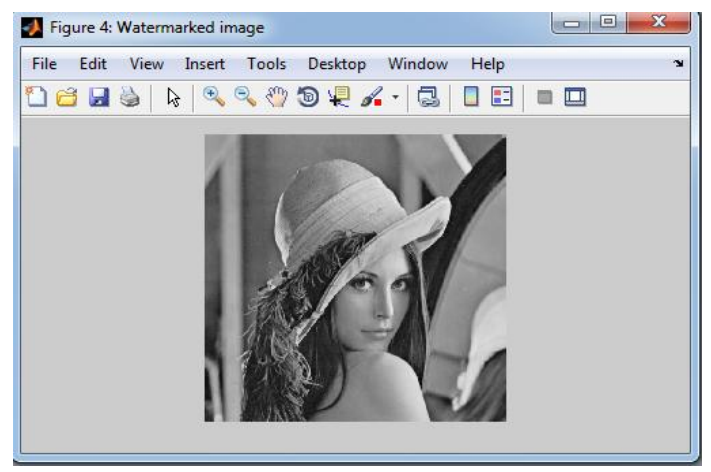

3) Watermarked Image

Table result .1 PSNR Values for DWT-SVD AND SWT-SVD FOR case A.

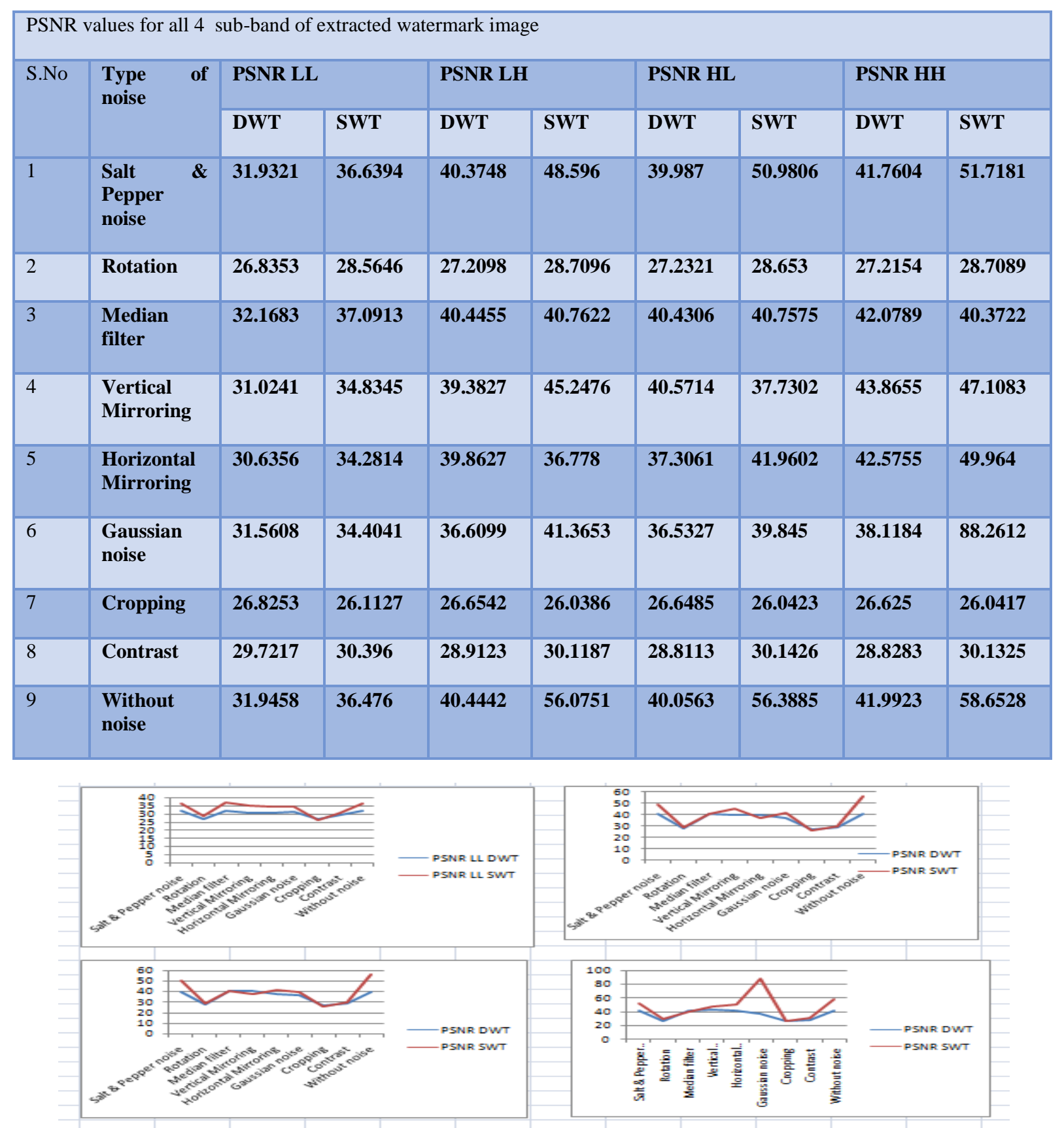

Fig.7Graphical results of PSNR for Case A. 
Case B) Considering input Cameraman image as cover image and college logo image as watermark image MATLAB results are as bellow DWT-SVD and SWT-SVD both results are given bellow

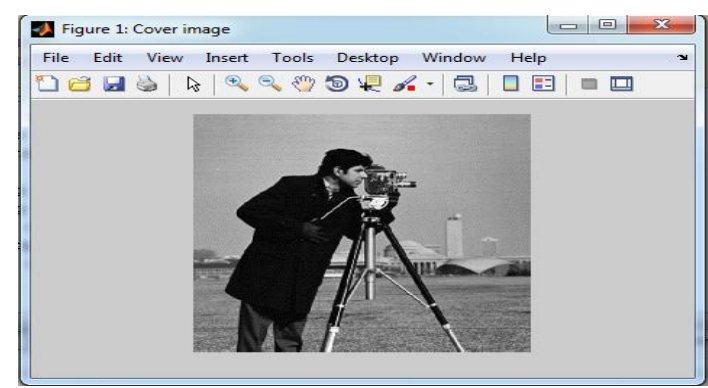

1) cover image : Camerman image

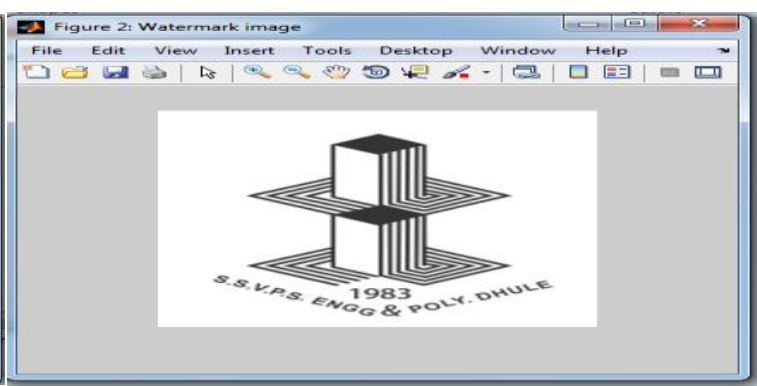

2) watermark image : college logo

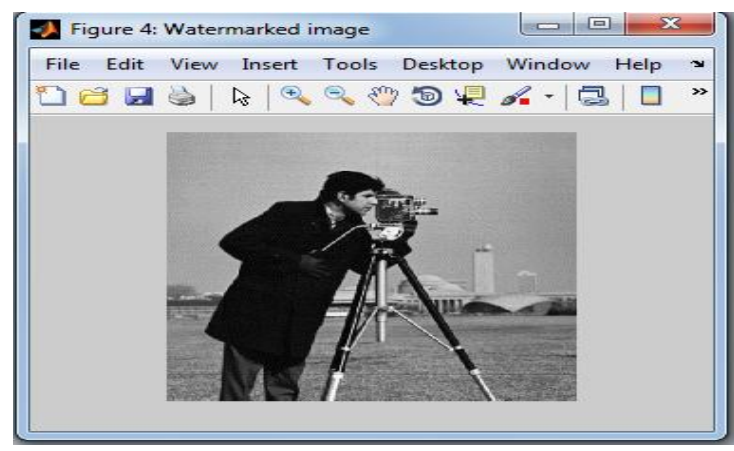

3) Watermarked image

Table result .2 PSNR Values for DWT-SVD AND SWT-SVD FOR case B.

\begin{tabular}{|c|c|c|c|c|c|c|c|c|c|}
\hline \multicolumn{10}{|c|}{ PSNR values for all 4 sub-band of extracted watermark image } \\
\hline \multirow[t]{2}{*}{ S.No } & \multirow[t]{2}{*}{ Type of noise } & \multicolumn{2}{|c|}{ PSNR LL } & \multicolumn{2}{|c|}{ PSNR LH } & \multicolumn{2}{|c|}{ PSNR HL } & \multicolumn{2}{|c|}{ PSNR HH } \\
\hline & & DWT & SWT & DWT & SWT & DWT & SWT & DWT & SWT \\
\hline 1 & Salt \& Pepper noise & 24.1483 & 28.66222 & 39.8564 & 46.7995 & 39.7349 & 43.9794 & 41.1972 & 43.3835 \\
\hline 2 & Rotation & 22.2388 & 24.9992 & 24.5986 & 26.485 & 24.5925 & 26.1606 & 24.4917 & 26.1836 \\
\hline 3 & Median filter & 24.1167 & 28.3469 & 37.4606 & 35.6765 & 37.4882 & 36.2419 & 38.3555 & 36.0218 \\
\hline 4 & Vertical Mirroring & 23.2596 & 27.1764 & 40.7921 & 35.6728 & 38.7077 & 37.0817 & 48.0338 & 34.6178 \\
\hline 5 & Horizontal Mirroring & 23.2226 & 27.1588 & 35.9818 & 35.0707 & 38.783 & 34.9363 & 37.8895 & 36.927 \\
\hline 6 & Gaussian noise & 23.7055 & 27.7006 & 32.5145 & 34.9826 & 32.4129 & 34.6323 & 32.9466 & 34.2563 \\
\hline 7 & Cropping & 28.2479 & 23.5558 & 23.5558 & 23.1082 & 24.4553 & 23.113 & 24.4077 & 23.1333 \\
\hline 8 & Contrast & 23.5943 & 25.5813 & 25.5813 & 25.5823 & 23.4447 & 25 & 23.4366 & 25.0584 \\
\hline 9 & Without noise & 24.1481 & 28.6703 & 28.67 & 45.0177 & 39.8255 & 45.8571 & 41.7627 & 43.5817 \\
\hline
\end{tabular}




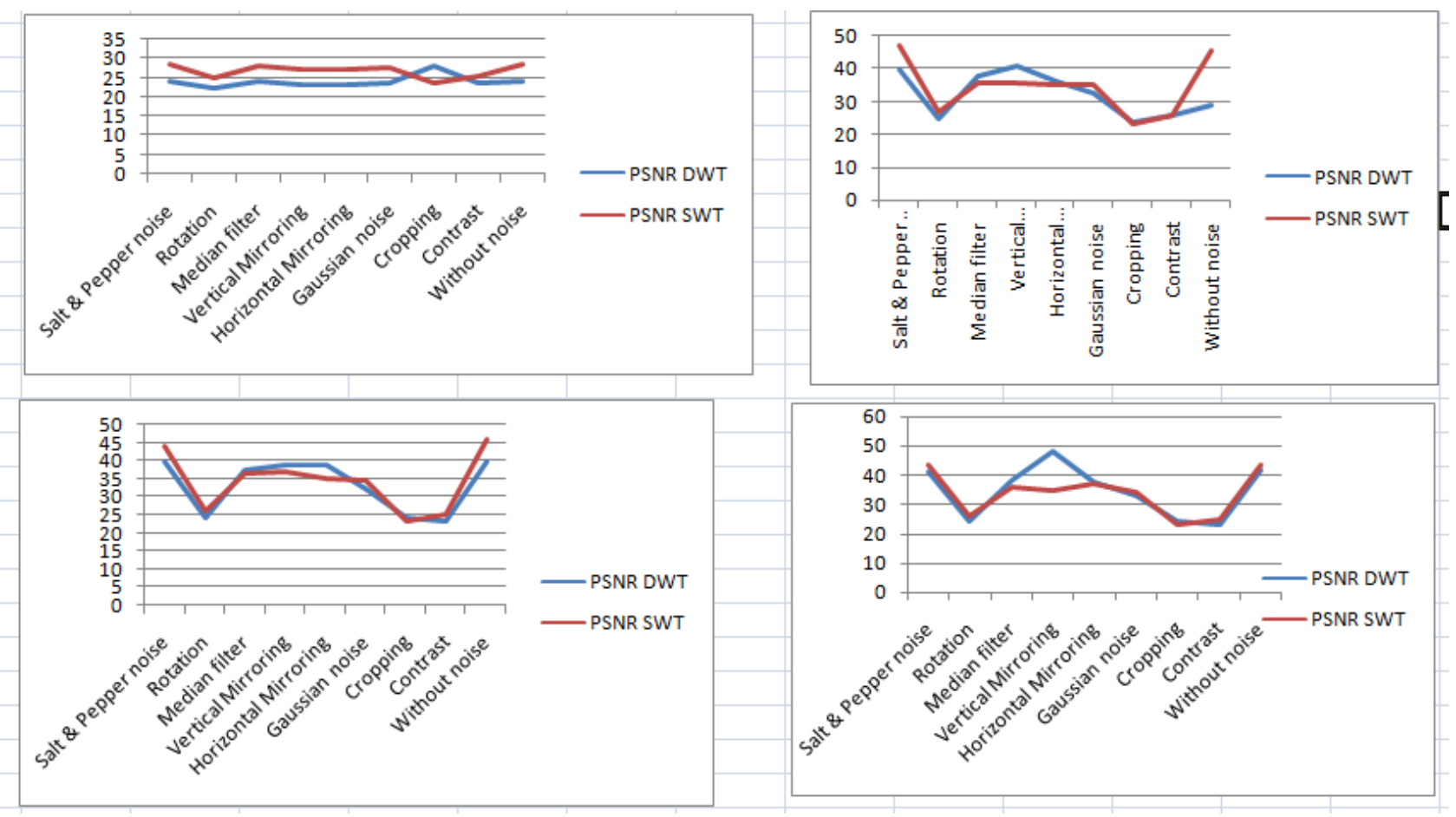

Fig.8 Graphical results of PSNR for Case B.

Case c) Considering input Tullips image as cover image and ICICI logo image as watermark image MATLAB results are as bellow DWT-SVD and SWT-SVD both results are given bellow

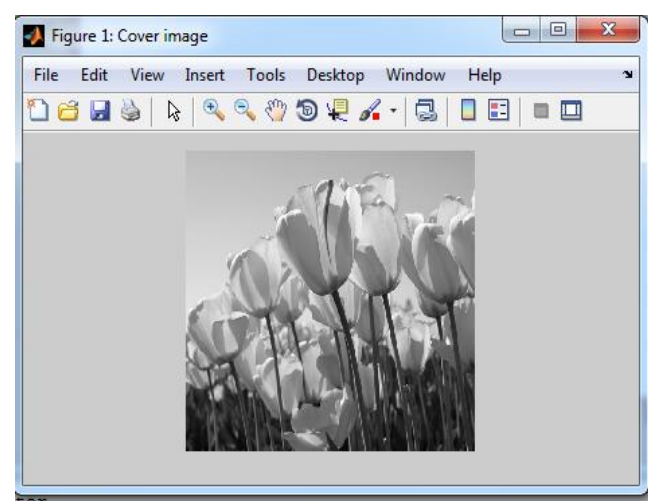

1) Cover image :

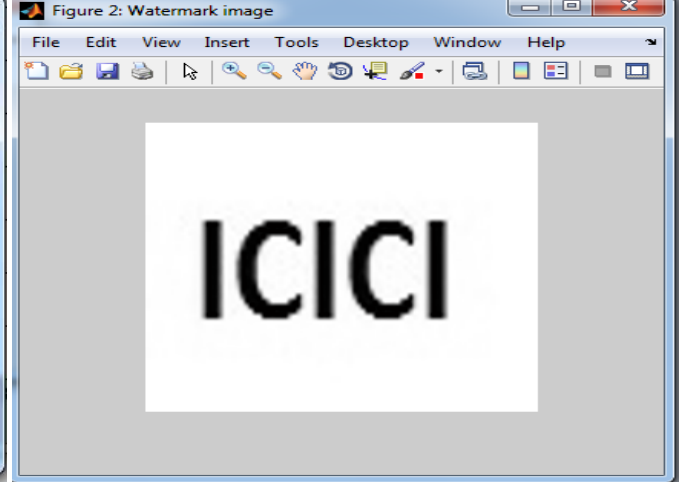

2) Watermark Image

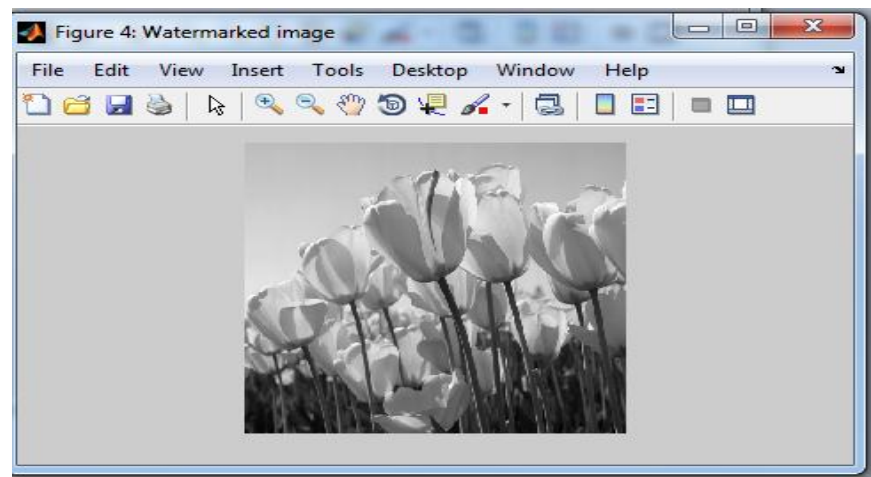

3) Watermarked Image 
Table Result .2 PSNR Values for DWT-SVD AND SWT-SVD FOR case C.

\begin{tabular}{|c|c|c|c|c|c|c|c|c|c|}
\hline \multicolumn{10}{|c|}{ PSNR values for all 4 sub-band of extracted watermark image } \\
\hline \multirow[t]{2}{*}{ Sr.No } & \multirow[t]{2}{*}{ Type of noise } & \multicolumn{2}{|c|}{ PSNR LL } & \multicolumn{2}{|c|}{ PSNR LH } & \multicolumn{2}{|c|}{ PSNR HL } & \multicolumn{2}{|c|}{ PSNR HH } \\
\hline & & DWT & SWT & DWT & SWT & DWT & SWT & DWT & SWT \\
\hline 1 & Salt \& Pepper noise & 24.0905 & 28.5703 & 40.9949 & 44.6078 & 40.6445 & 45.6057 & 42.1514 & 51.1027 \\
\hline 2 & Rotation & 18.1427 & 19.6822 & 18.2784 & 19.7524 & 18.2787 & 19.7752 & 18.2783 & 19.7859 \\
\hline 3 & Median filter & 24.1307 & 28.8865 & 40.6249 & 35.741 & 40.6727 & 35.7373 & 40.9241 & 36.6963 \\
\hline 4 & Vertical Mirroring & 27.9684 & 29.8788 & 42.2937 & 28.4368 & 42.0517 & 38.3005 & 39.198 & 48.7411 \\
\hline 5 & Horizontal Mirroring & 27.5232 & 30.0452 & 41.2452 & 30.3116 & 42.2849 & 42.3577 & 41.0419 & 36.2603 \\
\hline 6 & Gaussian noise & 24.1542 & 28.5452 & 37.3221 & 39.3659 & 27.7957 & 37.5458 & 38.3349 & 39.2603 \\
\hline 7 & Cropping & 30.6682 & 27.429 & 27.7942 & 24.9771 & 27.7957 & 25.0222 & 27.6592 & 25 \\
\hline 8 & Contrast & 23.1538 & 24.6573 & 23.222 & 24.6767 & 23.2224 & 25 & 23.2026 & 24.6813 \\
\hline 9 & Without noise & 24.0788 & 28.5853 & 40.5694 & 28.5853 & 40.5672 & 48.3511 & 42.1261 & 54.4602 \\
\hline
\end{tabular}

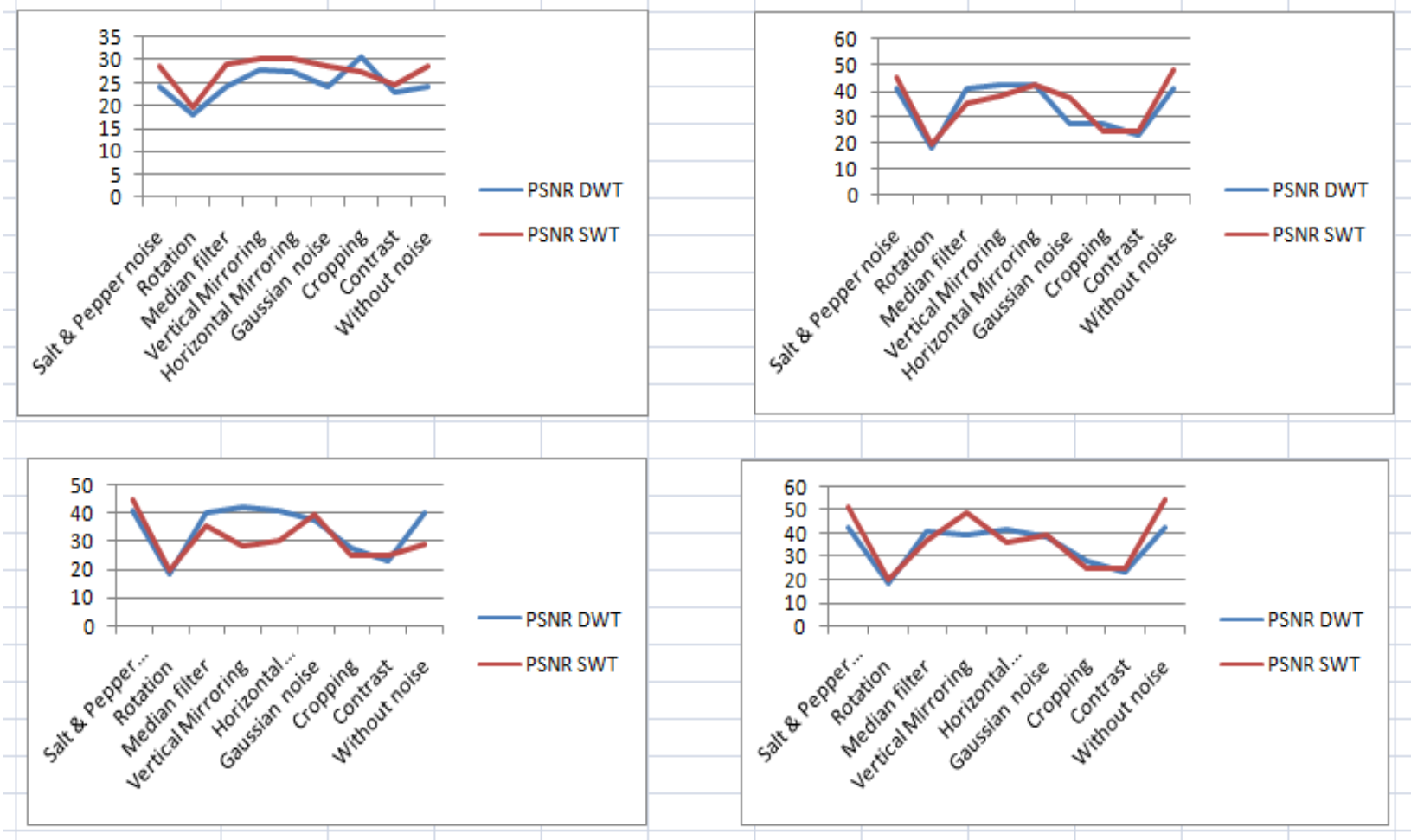

Fig.9 Graphical results of PSNR for Case C.

\section{CONCLUSION}

This paper present Digital image-watermarking technique based DWT-SVD and SWT-SVD where the watermark is embedded on the singular values of the cover image's SWT sub bands. The technique fully exploits the respective feature of these two transform domain method. Spatial frequency localization of SWT and SVD efficiently represents intrinsic algebraic properties of an image. Experiment results of the proposed technique have shown both the significant improvement in imperceptibility and the robustness under attacks quality of cover image is not degraded.by using SWTSVD.In SWD-SVD large size Watermarks has been used. Experimental results shows related PSNR Values Which shows SWT-SVD is gives better result than DWT-SVD. 


\section{REFERENCES}

[1] I.cox, J.killan,F.Leighton and T.shamoon,"secure spreadspectrum watermarking for Multimedia" IEEE Transactions on image processing ,vol.06,pp.16731687,1997

[2] D.Kundur and D.Hatzinakos,"A Robust Digital image watermarking method using wavelet based fusion", proceeding of international conference on image processing, IEEE Vol.01, pp.544-5471998

[3] F.Bartolini , M.Barni,V.Cappellini and A.Piva ,"mask Building for perceptually Hiding frequency Embedded Watermarks", Proceedings of international Conference on Image Processing , IEEE ,vol 01,pp 450-454,1999

[4] AsnaFurqan,Munish Kumar" Study and Analysis of Robust DWT-SVD Domain Based Digital Image Watermarking Technique Using MATLAB'IEEE, 2015 IEEE
[5] Dr.M.MohamedSathikand,S.S.Sujatha "Authentication of digital images by using Semi fragile watermarking Technique" vol 2pp 39-44,nov 2012

[6] Xiang Zhou., Duan X., and Wang D., "A Semi-fragile Watermark Scheme for Image Authentication", IEEE International Conference on Multimedia modeling, pp.374-377 (2004).

[7] Mehmet UtkuCelik, Gaurav Sharma and Ahmet Murat Tekalp "Hierachical watermarking for secure image Authentication with Localization"Vol.11,pp 585595,june 2002

[8] PrafulSaxena, ShanonGarg and Arpita Srivastava "DWTSVD Semi-Blind Image Watermarking Using High Frequency Band”April 28-29,2012

[9] Emir Ganic,AhmetM.Eskicioglu "Robust DWT-SVD Domain image watermarking :Embedding Data in all Frequencies" 